\title{
Guest editorial: Information and communications technology for development
}

\author{
Balaji Parthasarathy $\cdot$ Krithi Ramamritham
}

Published online: 17 April 2009

(C) Springer Science + Business Media, LLC 2009

This issue presents selected papers from the second international conference on Information and Communication Technologies and Development (ICTD2007) held in Bangalore from 15-16 December 2007. Following the enthusiastic reception to the first edition of the conference in Berkeley, California, in May 2006, the Bangalore conference built on the ICTD goal of providing a forum for scholarly work and practice in the field. Toward this goal, the conference solicited papers that were multi-disciplinary in scope and reported original research in a scientifically rigorous fashion (see http:// research.microsoft.com/en-us/um/india/events/ictd2007/cfp. $\mathrm{htm}$ ) with the expectation is that papers establish a clear relationship between ICTs and development.

To help potential authors come to grips with the diverse disciplines and methodologies that ICTD spans, Kentaro Toyama produced a note (see http://research.microsoft.com/ users/toyama/On Writing ICTD Research Papers.doc) on the standards against which ICTD papers should be judged. Toyama calls for novelty (including, but not limited to, a new thesis or invention, fresh data, innovative methodology, or first-time evaluations that also encompass negative results); applicability (solving an anticipated problem or question); and empirically grounded research (as opposed to papers that are primarily theoretical or speculative). $\mathrm{He}$

Balaji Parthasarathy and Krithi Ramamritham were the Program co-chairs of ICTD2007.

B. Parthasarathy $(\bowtie)$

International Institute of Information Technology,

Bangalore, India

e-mail: pbalaji@iiitb.ac.in

K. Ramamritham

Department of Computer Science and Engineering, Indian Institute of Technology Bombay,

Mumbai, India also emphasizes the importance of generalizability, replicability, and verifiability. Toyama's note prompted Jenna Burrell of the University of California, Berkeley, to respond with a call for a more interpretive approach to ICTD research, and for an explicit acknowledgement of the specifics of research contexts. In other words, she calls for an interrogation of the contexts in which using ICTs to overcome information asymmetries is meaningful. She also urges that the research characteristics identified by Toyama be broadened to include accuracy of variables and categories (instead of using those that are determined either a priori or exogenously), thick description of the factors influencing daily life at a site, and rigor that values multiple methods of arriving at conclusions. Many approaches and issues discussed in this debate are reflected in the papers that were published at ICTD 2007, four of which have been selected for this special issue

In the paper titled "AIR: Advancement through Interactive Radio", Sterling et al describe the rationale, design and implementation of a system to increase the status of women in developing communities. AIR gives female community radio listeners a means to respond to what they hear and to create content. It is designed on the assumption that direct interaction between women and community radio will have a positive influence on community radio programming, ultimately benefiting women and the larger community. During its ensuing deployment, the authors hope to critically examine the benefits attributable to AIR.

The authors of the second paper, Karla et al, are led by the observation that high illiteracy rates among the blind in developing countries is partly attributable to the lack of trained teachers and the difficulties faced in using traditional slate and stylus for writing Braille. We expect their paper, titled "Design of a Braille Writing Tutor to Combat Illiteracy", to serve as essential reading for those develop- 
ing new tools for the disabled. Not surprisingly, the paper points out that user involvement at both the design and testing stages is necessary for developing a successful tool.

The results of a qualitative study of computer-aided learning centers in schools catering primarily to very low income families in Southern India are presented by Pal et al, in "My Child Will Be Respected: Parental Perspectives on Computers and Education in Rural India." The paper presents many learnings, some anticipated and some not, from their interview and participant-observation based study. Its authors hope that this study will generate discussion on new perspectives on thinking about computers, parents and development.

"If the State provided free computer literacy, would it find takers? Evidence and propositions from the Akshaya project in India" is the title of the fourth and final paper in our selection. In it, author Pal adds to the already substantial literature on the Akshaya project from Kerala. The paper's focus is the project's e-literacy goal: one member of every household should be trained in the
Akshaya telecenters. Through his survey of 1,750 households in the experimental area of Malappuram district, and a comparison group in neighbouring Kozhikode district, Pal finds that overall participation in free e-literacy services among poor households remains low in spite of the wide publicity the project has received. Given this, we believe that the debate over the use of public funds for telecenter projects is bound to continue.

We hope that the papers published here stimulate further research and discussions on the topic of ICT for Development.

Balaji Parthasarathy is the ICICI Associate Professor at the International Institute of Information Technology, Bangalore.

Krithi Ramamritham is at the Indian Institute of Technology, Bombay, as the Vijay and Sita Vashee Chair Professor in the Department of Computer Science and Engineering. During the last few years he has been interested in the use of Information and Communication Technologies for creating tools aimed at socioeconomic development. 